\title{
Distinct Glucose-Lowering Mechanisms of Ipragliflozin Depending on Body Weight Changes
}

\author{
Eiji Kutoh $^{1,2,3} \cdot$ Teruma Murayama $^{1} \cdot$ Asuka Wada $^{2} \cdot$ Mitsuru Hirate $^{1}$
}

Published online: 31 October 2016

(c) The Author(s) 2016. This article is published with open access at Springerlink.com

\begin{abstract}
Background Sodium-glucose co-transporter 2 inhibitors have been shown to reduce body weight. However, little is known about whether a reduction in body weight affects glycemic and non-glycemic parameters.

Objectives The aim of this study was to investigate the link between the changes in body weight and those in metabolic parameters in drug-naïve subjects with type 2 diabetes mellitus (T2DM) receiving ipragliflozin monotherapy.

Methods Subjects received ipragliflozin monotherapy $25-50 \mathrm{mg} / \mathrm{day}$ for 3 months $(n=33)$. They were then divided into two groups: group L ('lost'; $n=17$ ) comprised patients who lost weight (change $[\Delta]$ in body mass index $[\mathrm{BMI}] \leq-0.75, p<0.00001$ ), and group $\mathrm{N}$ ("neutral'; $n=16$ ) comprised patients who did not lose weight $(\Delta \mathrm{BMI}>-0.75$, not significant $[\mathrm{NS}])$.

Results In these two groups, similar reductions were observed in glycated hemoglobin $\left(\mathrm{HbA}_{1 \mathrm{c}}\right.$ ) levels (group L: $9.76-8.02 \%, \quad p<0.00001 ;$ group $\mathrm{N}: 10.07-8.36 \%$, $p<0.0005$ ). Homeostasis model assessment (HOMA)-B levels increased in both groups, with inter-group differences $(p<0.05 ;+38.91$ vs. $+96.83 \%$ in group $\mathrm{L}$ and $\mathrm{N}$, respectively). However, some parameters showed distinct regulatory patterns. For instance, in group L, reductions were
\end{abstract}

Eiji Kutoh

ekuto@excite.com; ekuto-biomed@umin.ac.jp

1 Department of Clinical Research, Biomedical Center, Tokyo 1320034, Japan

2 Division of Diabetes and Endocrinology, Department of Internal Medicine, Gyoda General Hospital, Saitama, Japan

3 Division of Diabetes and Metabolism, Department of Internal Medicine, Higashitotsuka Memorial Hospital, Yokohama, Japan observed in HOMA-R $(-20.18 \%, p<0.04)$ and uric acid (UA; $-8.91 \%, p<0.02$ ) levels. Correlations were seen between the change in HOMA-R and those in fasting blood glucose (FBG) levels $(R=0.557, p<0.02)$. Non-significant increases in free fatty acid (FFA) levels and decreases in non-high-density lipoprotein cholesterol (non-HDL-C) or low-density lipoprotein cholesterol (LDL-C) levels were also noted. In group $\mathrm{N}$, reductions in FFA levels $(-17.07 \%$, $p<0.05)$ were observed, and negative correlations were seen between $\triangle$ HOMA-B and $\triangle \mathrm{FBG}(R=-0.4781$, $p<0.05)$ and between $\Delta$ FFA and $\Delta$ HOMA-B levels $(R=-0.4305, p<0.05)$. Non-significant increases in nonHDL-C and LDL-C levels were also noted. Inter-group differences existed between group $\mathrm{L}$ and group $\mathrm{N}$ in the changes in non-HDL-C and LDL-C levels (both $p<0.05$ ).

Conclusions These results indicate that ipragliflozin may possess distinct dual glucose-lowering mechanisms depending on body weight changes. Degrees of insulin resistance decrease in subjects who lose weight. Conversely, ipragliflozin reduces lipotoxicity (FFA levels), thereby activating beta-cell function, in subjects who do not lose weight. Similar glycemic efficacies were observed in both cases. In patients who lost weight, ipragliflozin was associated with improvements in the levels of metabolic parameters related to cardiovascular risk factors, including UA and atherogenic lipid levels (non-HDL-C and LDL-C) compared with those who did not lose weight.

\section{Key Points}

Distinct glucose-lowering mechanisms and effects on levels of atherogenic lipids or uric acid are observed depending on changes in body weight with ipragliflozin. 


\section{Introduction}

Sodium-glucose co-transporter 2 (SGLT-2) inhibitors are novel glucose-lowering agents that exert their therapeutic effects by facilitating glucose excretion through the kidneys [1-3]. This pharmacologically induced glycosuria causes physiological and adaptive responses in glucose homeostasis and other biomarkers (or metabolic parameters). For example, SGLT-2 inhibitors have also been shown to possess some non-glycemic benefits such as weight reduction, blood pressure control, diuretic action, renal protection, and reductions in levels of triglycerides (TG) and uric acid (UA) [4, 5]. Ipragliflozin is an SGLT-2 inhibitor that was recently launched in Japan [6, 7]. Similar glycemic and non-glycemic efficacies were reported with other SGLT-2 inhibitors [8, 9]. Via the simple mechanism of discarding glucose into the urine (correcting glucotoxicity) [10], ipragliflozin was shown to ameliorate impaired beta-cell function and insulin resistance [11]. Furthermore, preliminary reports indicated that ipragliflozin could regulate free fatty acid (FFA) levels, which might consequently influence lipotoxicity and thereby affect beta-cell function [12]. However, their mechanisms of action mean they are associated with higher incidences of certain adverse events, including genital mitotic infections, urinary tract infections, osmotic diuretic-related adverse events, and volume depletion-related adverse events [13]. Further, recent reports of potential SGLT-2 inhibitor-induced diabetic ketoacidosis (DKA) have raised concerns that this class of drugs might increase the risk of DKA, especially among patients receiving exogenous insulin [14]. SGLT2 inhibitors are currently used as add-on therapy to metformin or other drugs as part of dual or triple therapy. However, they could also be used as an alternative first-line option in patients with contraindications to or intolerance of metformin (see Kutoh et al. [11] and the references therein).

One of the most favorable non-glycemic efficacies of SGLT-2 inhibitors, including ipragliflozin, is their ability to reduce body weight $[4,11]$. However, little is known about whether a reduction in body weight affects glycemic and non-glycemic parameters. Preliminary results suggested that reductions in body weight were not associated with changes in glycemic parameters [11, 15]. This study was initiated to investigate the link between changes in body weight and changes in glycemic and non-glycemic parameters. It makes sense to perform this kind of study with monotherapy to eliminate the influence of other drugs as much as possible. As an initial step towards investigating this issue, we studied drug-naïve subjects with type 2 diabetes mellitus (T2DM) receiving ipragliflozin monotherapy and monitored any subsequent effects on glycemic and non-glycemic parameters in relation to changes in body weight.

\section{Subjects and Methods}

\subsection{Subjects}

Participants were newly diagnosed with T2DM (according to Japan Diabetes Society criteria [16]) or previously diagnosed but untreated. All subjects had received no regularly prescribed drugs for at least 6 months prior to study initiation. Subjects were excluded if they had clinically significant renal (creatinine $>1.5 \mathrm{mg} / \mathrm{dl}$ ) or liver (aspartate aminotransferase [AST]/alanine aminotransferase [ALT] >70/70 IU/l) dysfunction, a history of heart disorders, severe hypertension (blood pressure $>160 /$ $100 \mathrm{mmHg}$ ), type 1 diabetes mellitus (T1DM), or were pregnant. Subjects were outpatients recruited between July 2014 and January 2016 from the Department of Internal Medicine of Gyoda General Hospital (Saitama, Japan) and other hospitals with which the first author (EK) was associated. Subjects received ipragliflozin $25-50 \mathrm{mg} /$ day monotherapy. A total of 40 subjects were initially enrolled in this study; seven withdrew after experiencing adverse events or for other reasons and were excluded from data analysis. The final analysis included 33 subjects. Subjects were encouraged to follow previously described exercise and diet regimens [17, 18]. Subjects provided informed consent, and the study protocol was approved by the Ethical Committee/Institutional Review Board of Gyoda General Hospital. The study was conducted in accordance with the principles of good clinical practice.

\subsection{Measurements}

The primary endpoint was change in body mass index (BMI) from baseline to 3 months. The secondary endpoint included glycated hemoglobin $\left(\mathrm{HbA}_{1 \mathrm{c}}\right)$, fasting blood glucose (FBG), insulin, homeostasis model assessment (HOMA)-R and -B, TG, high-density lipoprotein cholesterol (HDL-C), non-HDL-C, low-density lipoprotein cholesterol (LDL-C), UA, and FFA. $\mathrm{HbA}_{1 \mathrm{c}}$ values were assessed using the National Glycoprotein Standardization Program (NGSP) [19, 20].

Blood was collected from patients in the fasting state before breakfast, and the standard technique was used to measure these parameters as described previously [18]. $\mathrm{HbA}_{1 \mathrm{c}}$ and FBG were measured once a month. Insulin was measured using a kit from Abbott Japan (Tokyo, Japan) at baseline and at the end of the study ( 3 months). Anti- 
glutamic acid decarboxylase (GAD) antibodies were measured in some patients to exclude those with T1DM (Mitsubishi BML, Tokyo, Japan). HOMA-R and HOMA-B were calculated as previously described [21]. Hepatic (AST, ALT, alkaline phosphatase [ALP], gamma-glutamyl transpeptidase $[\gamma-\mathrm{GTP}]$ ) and renal (blood urea nitrogen [BUN] and creatinine) functions were also monitored 1 month after initiating ipragliflozin. If any of these parameters had increased significantly, we discontinued ipragliflozin. Subjects were divided into two groups: group L ('lost', $n=17$ ) comprised patients who lost significant weight (change in BMI $[\Delta \mathrm{BMI}] \leq-0.75 ; p<0.00001$ ), and group $\mathrm{N}$ ('neutral', $n=16$ ) comprised patients who did not lose weight $(\Delta \mathrm{BMI}>-0.75 ; p$ value not significant [NS]). In group L, two subjects received ipragliflozin $25 \mathrm{mg} /$ day and 15 subjects received ipragliflozin $50 \mathrm{mg} /$ day. In group $\mathrm{N}$, two subjects received ipragliflozin $25 \mathrm{mg} /$ day and 14 subjects received ipragliflozin $50 \mathrm{mg} /$ day.

\subsection{Data Analyses}

We calculated change as 3-month values (post-therapy) minus baseline values (pre-therapy). When the data were normally distributed, we used a paired Student's $t$ test to analyze the changes in each group (intra-group differences). An unpaired Student's $t$ test was employed to compare baseline values in these two groups. We used the Wilcoxon signed-rank test to analyze data that were not normally distributed and analysis of covariance
(ANCOVA) to compare inter-group differences. We conducted simple regression analysis to analyze the correlations between changes in parameters. We conducted multiple regression analysis to identify any contributing factors for changes in BMI with ipragliflozin. The following independent variables (baseline levels) were used: age, $\mathrm{HbA}_{1 \mathrm{c}}$, FBG, HDL-C, TG, LDL-C, UA, HOMA-R, HOMA-B, and BMI. The results were expressed as mean \pm standard deviation (SD). Throughout the statistical analysis, $p<0.05$ was considered significant.

\section{Results}

\subsection{Effects on Glycemic and Non-Glycemic Parameters with Ipragliflozin (All Subjects)}

At 3 months, we observed significant reductions in FBG, $\mathrm{HbA}_{1 \mathrm{c}}$, BMI, HOMA-R, and UA levels and significant increases in HOMA-B levels. Little, if any, changes were noted in lipid parameters, including total cholesterol (TC), TG, HDL-C, non-HDL-C, and LDL-C levels (see Table 1 for values and statistical significance). Significant correlations were observed between $\triangle \mathrm{BMI}$ and changes in levels of TC, non-HDL-C, or LDL-C (Table 2). Conversely, and unexpectedly, no correlations were seen between $\triangle \mathrm{BMI}$ and changes in glycemic parameters (e.g., $\Delta \mathrm{HbA}_{1 \mathrm{c}}$ or $\Delta \mathrm{FBG}$; Table 2). We conducted multiple regression analysis to identify contributing factors for the changes in BMI with ipragliflozin. We used the following independent
Table 1 Baseline characteristics and changes in diabetic parameters with ipragliflozin (all subjects)

\begin{tabular}{|c|c|c|c|c|}
\hline Characteristics & Baseline & 3 months & $\%$ changes & $p$ values \\
\hline $\operatorname{Sex}(\mathrm{F} / \mathrm{M})$ & $5 / 28$ & & & \\
\hline Age (years) & $55.3 \pm 11.3$ & & & \\
\hline FBG (mg/dl) & $193.9 \pm 61.7$ & $150.0 \pm 39.7$ & -22.64 & $<0.0002$ \\
\hline $\mathrm{HbA}_{1 \mathrm{c}}(\%)$ & $9.90 \pm 2.02$ & $8.18 \pm 1.60$ & -17.37 & $<0.00001$ \\
\hline Insulin $(\mu \mathrm{U} / \mathrm{ml})$ & $7.05 \pm 3.82$ & $7.69 \pm 4.89$ & 9.07 & NS \\
\hline HOMA-R & $3.39 \pm 1.98$ & $2.76 \pm 1.77$ & -18.58 & $<0.01$ \\
\hline HOMA-B & $23.95 \pm 18.40$ & $38.85 \pm 31.79$ & 62.21 & $<0.001$ \\
\hline UA (mg/dl) & $5.56 \pm 1.46$ & $5.24 \pm 1.56$ & -5.75 & $<0.03$ \\
\hline BMI & $25.64 \pm 3.98$ & $25.00 \pm 4.01$ & -2.9 & $<0.0005$ \\
\hline FFA (eE/l) & $0.770 \pm 0.241$ & $0.752 \pm 0.350$ & -2.33 & NS \\
\hline $\mathrm{TC}(\mathrm{mg} / \mathrm{dl})$ & $218.0 \pm 39.9$ & $220.0 \pm 29.1$ & 0.91 & NS \\
\hline TG (mg/dl) & $235.2 \pm 224.1$ & $223.2 \pm 217.5$ & -5.1 & NS \\
\hline HDL-C (mg/dl) & $50.8 \pm 11.7$ & $52.9 \pm 13.1$ & 4.13 & NS \\
\hline Non-HDL-C (mg/dl) & $167.1 \pm 39.4$ & $167.0 \pm 30.8$ & -0.05 & NS \\
\hline LDL-C (mg/dl) & $137.3 \pm 38.6$ & $138.0 \pm 30.2$ & 0.5 & NS \\
\hline
\end{tabular}

$B M I$ body mass index, $F$ female, $F B G$ fasting blood glucose, $F F A$ free fatty acid, $H b A_{l c}$ glycated hemoglobin, $H D L-C$ high-density lipoprotein cholesterol, $H O M A-B / R$ homeostasis model assessment-B/R, $L D L$ $C$ low-density lipoprotein cholesterol, $M$ male, $N S$ not significant, $T C$ total cholesterol, $T G$ triglyceride, $U A$ uric acid 
Table 2 Correlations between change in body weight and change glycemic and non-glycemic parameters. Simple regression analysis was performed between the indicated parameters

\begin{tabular}{lll}
\hline Parameters & $R$ & $p$ values \\
\hline$\Delta$ BMI vs. $\Delta$ FBG & -0.0109 & NS \\
vs. $\Delta$ HbA $_{1 \mathrm{c}}$ & 0.1836 & NS \\
vs. $\Delta$ insulin & 0.2745 & NS \\
vs. $\Delta$ HOMA-R & 0.1446 & NS \\
vs. $\Delta$ HOMA-B & 0.1302 & NS \\
vs. $\Delta$ UA & 0.2229 & NS \\
vs. $\Delta$ FFA & 0.0318 & NS \\
vs. $\Delta$ TC & 0.4672 & $<0.01$ \\
vs. $\Delta$ TG & 0.1522 & NS \\
vs. $\Delta$ HDL-C & 0.0794 & NS \\
vs. $\Delta$ non-HDL-C & 0.5084 & $<0.005$ \\
vs. LDL-C & 0.3731 & $<0.05$ \\
\hline
\end{tabular}

$B M I$ body mass index, $F B G$ fasting blood glucose, $F F A$ free fatty acid, $H b A_{l c}$ glycated hemoglobin, $H D L-C$ high-density lipoprotein cholesterol, HOMA-B/R homeostasis model assessment-B/R, $L D L-C$ low-density lipoprotein cholesterol, $N S$ not significant, $T C$ total cholesterol, $T G$ triglyceride, $U A$ uric acid

variables: age, $\mathrm{HbA}_{1 \mathrm{c}}$, FBG, BMI, HDL-C, TG, LDL-C, UA, insulin, HOMA-R, HOMA-B, and BMI. However, no significant contributing factors were identified (results not shown).

\subsection{Differential Regulations of Diabetic Parameters with Ipragliflozin Depending on Body Weight Changes}

Baseline parameter characteristics were similar between the groups, and no statistically significant differences were noted, except that BMI and lipid (TC, TG, HDL-C, nonHDL-C, LDL-C) levels tended to be higher in group $\mathrm{L}$ than in group $\mathrm{N}$ (Table 3). Reductions in $\mathrm{HbA}_{1 \mathrm{c}}$ and $\mathrm{FBG}$ levels were similar for the two groups (Table 4). HOMA-B levels increased in both groups, with significant inter-group differences (Fig. 1a, $p<0.05$; +96.83 vs. $+38.91 \%$ in group $\mathrm{N}$ and group $\mathrm{L}$, respectively).

However, other parameters showed distinct regulatory patterns. In group L, we observed significant reductions in HOMA-R $(-20.18 \% ; p<0.04)$ and UA $(-8.91 \%$; $p<0.02)$ levels. Insignificant increases in FFAs and decreases in non-HDL-C and LDL-C levels were noted (see Table 4). In group $\mathrm{N}$, significant reductions in FFA levels $(-17.07 \% ; p<0.05)$ and insignificant increases in non-HDL-C and LDL-C levels were observed (see Table 4). Significant inter-group differences existed in the changes in non-HDL-C and LDL-C levels (both $p<0.05$, see Fig. 1b, c).
Table 3 Baseline characteristics of metabolic parameters between group L and group $\mathrm{N}$

\begin{tabular}{|c|c|c|c|}
\hline Characteristics & Group N & Group L & $p$ values \\
\hline $\operatorname{Sex}(F / M)$ & $2 / 14$ & $3 / 14$ & NS \\
\hline Age (years) & $53.1 \pm 11.5$ & $57.3 \pm 11.1$ & NS \\
\hline FBG $(\mathrm{mg} / \mathrm{dl})$ & $201.1 \pm 63.5$ & $187.9 \pm 61.4$ & NS \\
\hline $\mathrm{HbA}_{1 \mathrm{c}}(\%)$ & $10.07 \pm 1.85$ & $9.76 \pm 2.20$ & NS \\
\hline Insulin $(\mu \mathrm{U} / \mathrm{ml})$ & $6.84 \pm 3.74$ & $7.23 \pm 3.98$ & NS \\
\hline HOMA-R & $3.46 \pm 1.97$ & $3.32 \pm 2.04$ & NS \\
\hline HOMA-B & $21.18 \pm 15.85$ & $26.26 \pm 20.45$ & NS \\
\hline $\mathrm{UA}(\mathrm{mg} / \mathrm{dl})$ & $5.51 \pm 1.20$ & $5.61 \pm 1.68$ & NS \\
\hline BMI & $25.29 \pm 4.91$ & $25.94 \pm 3.12$ & 0.064 \\
\hline FFA $(e E q / l)$ & $0.808 \pm 0.225$ & $0.739 \pm 0.255$ & NS \\
\hline $\mathrm{TC}(\mathrm{mg} / \mathrm{dl})$ & $204.6 \pm 36.6$ & $228.3 \pm 40.3$ & NS \\
\hline $\mathrm{TG}(\mathrm{mg} / \mathrm{dl})$ & $198.0 \pm 186.6$ & $264.2 \pm 250.8$ & NS \\
\hline HDL-C (mg/dl) & $48.7 \pm 12.2$ & $52.4 \pm 11.4$ & NS \\
\hline Non-HDL-C (mg/dl) & $155.9 \pm 32.0$ & $175.9 \pm 43.2$ & NS \\
\hline LDL-C (mg/dl) & $131.4 \pm 34.1$ & $142.0 \pm 42.1$ & NS \\
\hline
\end{tabular}

$B M I$ body mass index, $F$ female, $F B G$ fasting blood glucose, $F F A$ free fatty acid, $H b A_{l c}$ glycated hemoglobin, $H D L-C$ high-density lipoprotein cholesterol, HOMA-B/R homeostasis model assessment-B/ $\mathrm{R}, L D L-C$ low-density lipoprotein cholesterol, $M$ male, $N S$ not significant, $T C$ total cholesterol, $T G$ triglyceride, $U A$ uric acid

\subsection{Modulation of Insulin Resistance and Beta-Cell Function in the Glycemic Efficacy of Ipragliflozin Depending on Body Weight Changes}

Our next question was whether glucose level reductions (assessed with FBG) correlated with changes in insulin resistance (assessed via HOMA-R) and/or beta-cell function (assessed via HOMA-B) according to changes in body weight (assessed with BMI). For this purpose, we conducted simple regression analysis of changes in these parameters between group L and group N. Significant correlations were observed between $\triangle$ HOMA-R and $\triangle F B G$ in group $\mathrm{L}$ (Table 5). Conversely, significant negative correlations were seen between $\triangle$ HOMA-B and $\triangle F B G$ and between $\triangle F F A$ and $\Delta$ HOMA-B in group $N$ (Table 5). No correlations were noted between other parameters (results not shown).

\section{Discussion}

\subsection{Link Between Body Weight Changes and Glycemic Efficacies with Ipragliflozin}

One of the most notable effects of ipragliflozin is the reduction in body weight (Table 1). This is similar to other SGLT-2 inhibitors [4]. Many drugs used in the treatment of 
Table 4 Changes in glycemic and non-glycemic parameters with ipragliflozin in two groups of subjects with distinct body weight changes

\begin{tabular}{|c|c|c|c|c|c|c|c|c|}
\hline \multirow[t]{2}{*}{ Parameters } & \multicolumn{4}{|l|}{ Group L } & \multicolumn{4}{|l|}{ Group N } \\
\hline & Baseline & 3 months & $\%$ changes & $p$ values & Baseline & 3 months & $\%$ changes & $p$ values \\
\hline $\operatorname{Sex}(\mathrm{F} / \mathrm{M})$ & $3 / 14$ & & & & $2 / 14$ & & & \\
\hline Age (years) & $57.3 \pm 11.1$ & & & & $53.1 \pm 11.5$ & & & \\
\hline BMI & $25.94 \pm 3.12$ & $24.66 \pm 3.10$ & -4.93 & $<0.00001$ & $25.29 \pm 4.91$ & $25.2 \pm 4.97$ & -0.35 & NS \\
\hline FBG (mg/dl) & $187.9 \pm 61.4$ & $152.2 \pm 47.4$ & -18.99 & $<0.04$ & $201.1 \pm 63.5$ & $147.4 \pm 28.6$ & -26.7 & $<0.0002$ \\
\hline $\mathrm{HbA}_{1 \mathrm{c}}(\%)$ & $9.76 \pm 2.20$ & $8.02 \pm 1.88$ & -17.82 & $<0.00001$ & $10.07 \pm 1.85$ & $8.36 \pm 1.23$ & -16.98 & $<0.0005$ \\
\hline Insulin $(\mu \mathrm{U} / \mathrm{ml})$ & $7.23 \pm 3.98$ & $7.22 \pm 3.91$ & -0.13 & NS & $6.84 \pm 3.74$ & $8.25 \pm 5.96$ & 20.61 & NS \\
\hline HOMA-R & $3.32 \pm 2.04$ & $2.65 \pm 1.66$ & -20.18 & $<0.04$ & $3.46 \pm 1.97$ & $2.90 \pm 1.94$ & -16.18 & NS \\
\hline HOMA-B & $26.26 \pm 20.45$ & $36.48 \pm 24.78$ & 38.91 & $<0.05$ & $21.18 \pm 15.85$ & $41.69 \pm 39.34$ & 96.83 & $<0.002$ \\
\hline $\mathrm{UA}(\mathrm{mg} / \mathrm{dl})$ & $5.61 \pm 1.68$ & $5.11 \pm 1.47$ & -8.91 & $<0.02$ & $5.51 \pm 1.20$ & $5.35 \pm 1.72$ & -2.9 & NS \\
\hline FFA (eEq/l) & $0.739 \pm 0.255$ & $0.820 \pm 0.422$ & 10.96 & NS & $0.808 \pm 0.225$ & $0.670 \pm 0.225$ & -17.07 & $<0.05$ \\
\hline $\mathrm{TC}(\mathrm{mg} / \mathrm{dl})$ & $228.3 \pm 40.3$ & $221.7 \pm 33.6$ & -2.89 & NS & $204.6 \pm 36.6$ & $217.7 \pm 23.1$ & 6.4 & NS \\
\hline TG (mg/dl) & $264.2 \pm 250.8$ & $252.0 \pm 272.7$ & -4.61 & NS & $198.0 \pm 186.6$ & $186.1 \pm 113.7$ & -6.01 & NS \\
\hline HDL-C (mg/dl) & $52.4 \pm 11.4$ & $53.8 \pm 13.3$ & 2.67 & NS & $48.7 \pm 12.2$ & $51.8 \pm 13.2$ & 6.36 & NS \\
\hline Non-HDL-C (mg/dl) & $175.9 \pm 43.2$ & $167.8 \pm 37.1$ & -4.6 & NS & $155.9 \pm 32.0$ & $165.9 \pm 21.5$ & 6.41 & NS \\
\hline LDL-C (mg/dl) & $142.0 \pm 42.1$ & $133.6 \pm 36.9$ & -5.91 & NS & $131.4 \pm 34.1$ & $143.6 \pm 18.4$ & 9.28 & NS \\
\hline
\end{tabular}

$B M I$ body mass index, $F$ female, $F B G$ fasting blood glucose, $F F A$ free fatty acid, $H b A_{l c}$ glycated hemoglobin, $H D L-C$ high-density lipoprotein cholesterol, HOMA-B/R homeostasis model assessment-B/R, $L D L-C$ low-density lipoprotein cholesterol, $M$ male, $N S$ not significant, TC total cholesterol, $T G$ triglyceride, $U A$ uric acid

diabetes, such as insulin, sulfonylureas, and thiazolidinediones, cause weight gain. Therefore, drugs that have neutral effects on or that can reduce body weight are particularly important. A recent study showed that the majority of the reduction in weight with ipragliflozin was due to the loss of body fat mass (abdominal and subcutaneous fat), and changes in lean body mass were minimal $[22,23]$. In an effort to identify factors that contributed to the reductions in body weight with ipragliflozin, we conducted multiple regression analysis using several glycemic and non-glycemic factors as independent variables (see the Sect. 2). However, no significant factors were identified in the test we ran. Unexpectedly, no correlations existed between change in body weight (assessed with BMI) and changes in glycemic parameters (FBG and $\mathrm{HbA}_{1 \mathrm{c}}$; Table 2). This conclusion was supported by another analysis in which the subjects were divided into two groups (group L and group N). Similar reductions in glycemic parameters were seen in these two groups (FBG and $\mathrm{HbA}_{1 \mathrm{c}}$; Table 4).

The mechanism of body weight reduction with SGLT-2 inhibitors is via increased glucosuria (discarding calories into urine) as expected. This mechanism also influences other metabolic parameters [4]: blood pressure lowers via osmotic diuretic actions [5]; serum UA levels decrease via alterations in UA transport activity (GLUT9 isoform 2) in renal tubules because of increased glucosuria [24]; and TG levels decrease via increased lipolysis [22]. Interestingly, as shown in this study, this drug can have good glycemic efficacy in those who do not lose weight (Table 4). Why do such populations still exhibit good glycemic efficacies? One potential explanation is that, in patients who do not lose weight with ipragliflozin, beta-cell function is activated and thus the physiological effectiveness of insulin (which is a lipogenic hormone) is restored, thereby offsetting the decrease of body weight via increased glucosuria (Fig. 2). Alternatively, these patients may possess novel glucose-lowering mechanisms other than enhanced urinary glucose excretion with this drug.

\subsection{Link Between Body Weight Changes and Non- Glycemic Efficacies with Ipragliflozin}

Based on our results, it is plausible that patients who lose weight while receiving ipragliflozin may have more advantages than those who do not in terms of some nonglycemic parameters (e.g., UA, LDL-C, or non-HDL-C), though similar glycemic efficacies were observed regardless of changes in body weight (Table 4; Fig. 1a-c). These parameters, including elevated levels of UA, LDL-C, and non-HDL-C, were known risk factors for cardiovascular (CV) diseases. The recently reported CV outcome trial, EMPA-REG OUTCOME, demonstrated that empagliflozin significantly reduced CV morbidity and mortality in subjects with T2DM and at high CV risk [25]. An understanding of the mechanisms of these favorable CV effects would be of significant interest, as would identifying the characteristics of patients who benefit from this drug. Our 

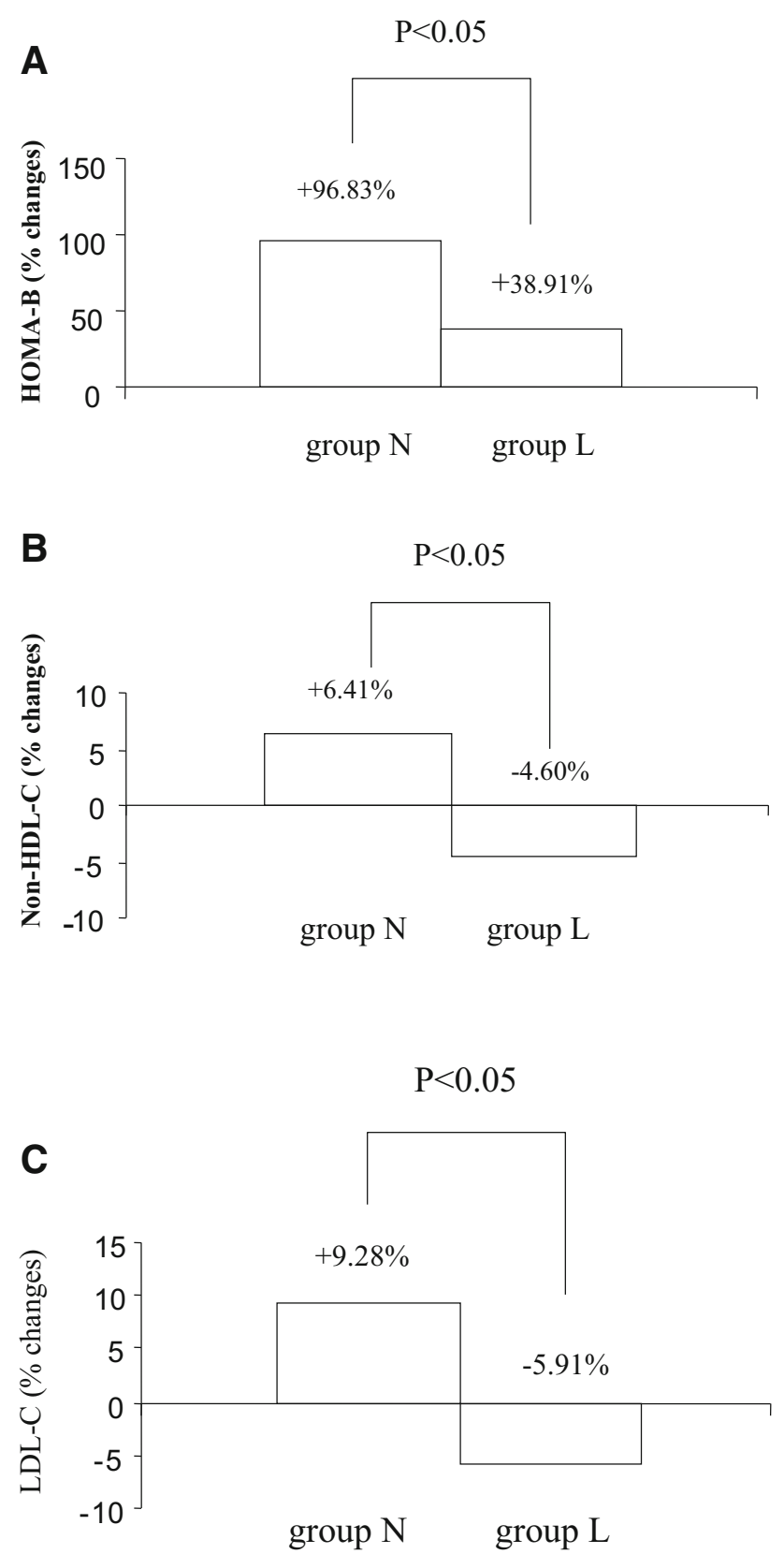

Fig. 1 Differential effects on metabolic parameters with ipragliflozin in subjects with distinct body weight changes. Analysis of covariance was performed to analyze the inter-group differences on the reductions between group $\mathrm{L}$ and group $\mathrm{N}$. a homeostasis model assessment-B, b non-high-density lipoprotein cholesterol. c Lowdensity lipoprotein cholesterol

hypothesis is that patients who lose weight have better CVrelated profiles (e.g., reductions in levels of UA, non-HDL$\mathrm{C}$, and LDL-C as we have shown; Table 4). Sub-analysis of the subjects in terms of weight changes in EMPA-REG may challenge this hypothesis. Finally, whether or not other SGLT-2 inhibitors, including ipragliflozin, have similar CV benefits remains to be seen.
Table 5 Correlations between the changes of glycemic parameters and those of insulin resistance (HOMA-R) and beta-cell functions (HOMA-B). Simple regression analysis was performed between the indicated parameters in group $\mathrm{L}$ and group $\mathrm{N}$

\begin{tabular}{lll}
\hline & $R$ & $p$ values \\
\hline Group L & & \\
$\Delta$ HOMA-R vs. $\Delta$ FBG & 0.557 & $<0.02$ \\
Group N & & \\
$\Delta$ HOMA-B vs. $\Delta$ FBG & -0.4781 & $<0.05$ \\
$\Delta$ FFA vs. $\Delta$ HOMA-B & -0.4305 & $<0.05$ \\
\hline
\end{tabular}

$F B G$ fasting blood glucose, $H O M A-B / R$ homeostasis model assessment-B/R, $\Delta$ indicates change

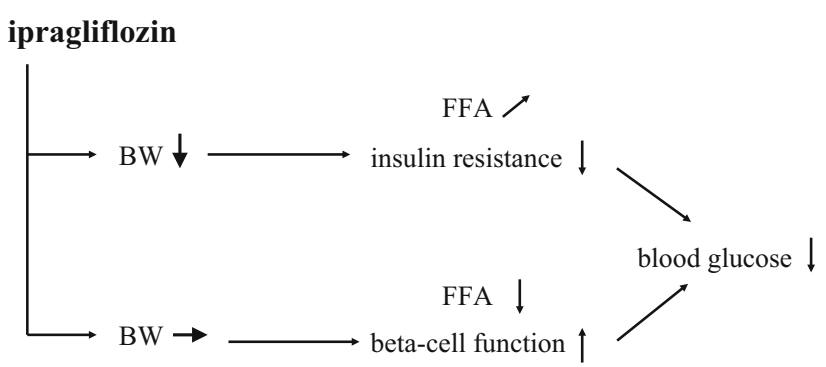

Fig. 2 Schematic representation of glycemic efficacy of ipragliflozin depending on changes in body weight. $B W$ body weight, $F F A$ free fatty acid, $\uparrow$ and $\downarrow$ indicate increase and decrease, respectively

\subsection{Modulation of Insulin Resistance and Beta-Cell Function with Ipragliflozin}

FFAs have been hypothesized to be the underlying cause and/or consequence of impaired beta-cell function, called 'lipotoxicity' [26, 27]. FFA levels significantly decreased in group $\mathrm{N}$ but tended to increase in group L (Table 4). Due to their mechanisms of action, SGLT2 inhibitors shift substrate utilization from carbohydrates to lipids. Thus, presumably, patients who lose weight have elevated FFA levels via decreased TG levels [22]. On the other hand, the mechanism by which ipragliflozin lowers high FFA levels in patients who do not lose weight remains to be investigated.

It appears that the glycemic efficacy of ipragliflozin is determined by the balance of its ability to modulate insulin resistance and beta-cell function depending on changes in body weight. Briefly, in group L, significant reductions in insulin resistance (HOMA-R) were seen (Table 4) and changes in HOMA-R levels were significantly correlated to changes in FBG (Table 5). Conversely, in group $\mathrm{N}$, we observed significant reductions in FFA levels (lipotoxicity) and higher degrees of increases in beta-cell function (HOMA-B) compared with patients who lost weight 
(Table 4; Fig. 1a). Indeed, significant negative correlations between changes in FFA and changes in HOMA-B, or between changes in HOMA-B and changes in FBG levels were seen (Table 5) in this population. Taken together, these results indicate ipragliflozin may have two distinct glucose-lowering mechanisms: reducing insulin resistance via weight loss (group L) and activating beta-cell function by reducing lipotoxicity (FFA levels; group N) (Fig. 2). To this end, it should be noted that the high baseline HOMA-R levels in these subjects may indicate the presence of nonalcoholic fatty liver disease (NAFLD) [28], and the effects of ipragliflozin on NAFLD are yet to be investigated.

\subsection{Limitations and Future Perspectives}

This study has a number of limitations. It is an observational (but prospective) study with a small number of subjects and a short duration. However, one can assume that the observed changes were exclusively caused by the tested drug based on the study design (monotherapy with drug-naïve subjects). Further randomized double-blind placebo-controlled studies over a longer period and with more subjects will be required to strengthen the findings from this study.

Another limitation is that the sex ratio (female/male) of the subjects was unbalanced (Table 1). More female subjects should be included to enable more solid conclusions. Furthermore, other important parameters (e.g., ketone bodies) that may be regulated by SGLT-2 inhibitors need to be measured in future studies. We are currently undertaking identical research with canagliflozin $100 \mathrm{mg} /$ day monotherapy in drug-naïve subjects with T2DM. These results will clarify whether the observed phenomenon in this work is a drug effect (specific for ipragliflozin) or a class effect of SGLT-2 inhibitors.

\section{Conclusions}

The results of this study add the following novel information to our current knowledge: ipragliflozin may possess distinct dual glucose-lowering mechanisms depending on body weight changes. Degrees of insulin resistance decrease in subjects who lose weight. Conversely, this drug reduces lipotoxicity (FFA levels) in subjects who do not lose weight, thereby activating beta-cell function. Similar glycemic efficacies were observed in these two populations. Furthermore, in patients who lose weight, ipragliflozin is associated with improvements in the levels of metabolic parameters related to $\mathrm{CV}$ risk factors, including UA and atherogenic lipids (non-HDL-C and LDL-C) compared with patients who do not lose weight.
Acknowledgements The authors thank Drs. Takashi Suzuki, Sayaka Terayama, Takeo Kikkawa, Mitsuru Nagao, and Hiroshi Kawashima for discussions and useful comments.

\section{Compliance with Ethical Standards}

Conflict of interest Eiji Kutoh, Teruma Murayama, Asuka Wada, and Mitsuru Hirate have no conflicts of interests that are directly relevant to the content of this manuscript.

Ethical Approval All subjects provided informed consent. The studies were conducted in accordance with the Declaration of Helsinki and International Conference on Harmonisation Good Clinical Practice (ICH GCP) guidelines.

Author Contributions EK participated in the design of the study and data acquisition, performed the statistical analysis, and drafted the manuscript. TM, MH, and AW made substantial contributions to the conception and design of the study and helped draft the manuscript. All authors read and approved the final manuscript.

Funding No sources of funding were used to conduct this study or prepare this manuscript.

Open Access This article is distributed under the terms of the Creative Commons Attribution-NonCommercial 4.0 International License (http://creativecommons.org/licenses/by-nc/4.0/), which permits any noncommercial use, distribution, and reproduction in any medium, provided you give appropriate credit to the original author(s) and the source, provide a link to the Creative Commons license, and indicate if changes were made.

\section{References}

1. Abdul-Ghani MA, Norton L, Defronzo RA. Role of sodiumglucose cotransporter 2 (SGLT 2) inhibitors in the treatment of type 2 diabetes. Endocr Rev. 2011;32(4):515-31.

2. Whalen K, Miller S, Onge ES. The role of sodium-glucose cotransporter 2 inhibitors in the treatment of type 2 diabetes. Clin Ther. 2015;37(6):1150-66.

3. DeFronzo RA, Davidson JA, Del Prato S. The role of the kidneys in glucose homeostasis: a new path towards normalizing glycaemia. Diabetes Obes Metab. 2012;14(1):5-14.

4. Scheen AJ, Paquot N. Metabolic effects of SGLT-2 inhibitors beyond increased glucosuria: a review of the clinical evidence. Diabetes Metab. 2014;40(6 Suppl 1):S4-11.

5. Oliva RV, Bakris GL. Blood pressure effects of sodium-glucose co-transport 2 (SGLT2) inhibitors. J Am Soc Hypertens. 2014;8(5):330-9.

6. Hedrington MS, Davis SN. Ipragliflozin, a sodium-glucose cotransporter 2 inhibitor, in the treatment of type 2 diabetes. Expert Opin Drug Metab Toxicol. 2015;11(4):613-23.

7. Poole RM, Dungo RT. Ipragliflozin: first global approval. Drugs. 2014;74(5):611-7.

8. Kurosaki E, Ogasawara H. Ipragliflozin and other sodium-glucose cotransporter-2 (SGLT2) inhibitors in the treatment of type 2 diabetes: preclinical and clinical data. Pharmacol Ther. 2013;139(1):51-9.

9. Kashiwagi A, Kazuta K, Yoshida S, Nagase I. Randomized, placebo-controlled, double-blind glycemic control trial of novel sodium-dependent glucose cotransporter 2 inhibitor ipragliflozin in Japanese patients with type 2 diabetes mellitus. J Diabetes Investig. 2014;5(4):382-91. 
10. Takahara M, Shiraiwa T, Matsuoka TA, Katakami N, Shimomura I. Ameliorated pancreatic $\beta$ cell dysfunction in type 2 diabetic patients treated with a sodium-glucose cotransporter 2 inhibitor ipragliflozin. Endocr J. 2015;62(1):77-86.

11. Kutoh E, Wada A, Murayama T, Hirate M. Ipragliflozin as an initial therapy in drug naïve subjects with type 2 diabetes. Drug Res (Stuttg). 2016;66(7):345-50.

12. Kutoh E, Wada A, Hirate M. Distinct glucose lowering mechanisms of ipragliflozin depending on body weight change. American Diabetes Association Scientific Meeting 2016 [1186-P].

13. Halimi $S$, Vergès $B$. Adverse effects and safety of SGLT-2 inhibitors. Diabetes Metab. 2014;40(6 Suppl 1):S28-34.

14. Handelsman Y, Henry RR, Bloomgarden ZT, Dagogo-Jack S, DeFronzo RA, Einhorn D, Ferrannini E, Fonseca VA, Garber AJ, Grunberger G, LeRoith D, Umpierrez GE, Weir MR. American Association of Clinical Endocrinologists and American College of Endocrinology position statement on the association of SGLT2 inhibitors and diabetic ketoacidosis. Endocr Pract. 2016;22(6):753-62.

15. Kashiwagi A, Yoshida S, Nakamura I, Kazuta K, Ueyama E, Takahashi H, Satomi H, Kosakai Y, Kawamuki K. Efficacy and safety of ipragliflozin in Japanese patients with type 2 diabetes stratified by body mass index: a subgroup analysis of five randomized clinical trials. J Diabetes Investig. 2016;7(4):544-54. doi:10.1111/jdi.12471.

16. Committee of the Japan Diabetes Society on the diagnostic criteria of diabetes mellitus. Report of the committee on the classification and diagnostic criteria of diabetes mellitus. J Diabetes Investig. 2010;1(5):212-228.

17. Ma Y, Olendzki BC, Merriam PA, Chiriboga DE, Culver AL, Li W, Hébert JR, Ockene IS, Griffith JA, Pagoto SL. A randomized clinical trial comparing low-glycemic index versus ADA dietary education among individuals with type 2 diabetes. Nutrition. 2008;24(1):45-56.

18. Kutoh E, Kaneoka N, Hirate M. Alogliptin: a new dipeptidyl peptidase-4 inhibitor with potential anti-atherogenic properties. Endocr Res. 2015;40(2):88-96.

19. Little RR, Rohlfing CL, Wiedmeyer HM, Myers GL, Sacks DB. Goldstein DE; NGSP Steering Committee. The national glycohemoglobin standardization program: a five-year progress report. Clin Chem. 2001;47(11):1985-92.

20. Miedema K. Towards worldwide standardisation of HbA1c determination. Diabetologia. 2004;47(7):1143-8.

21. Matthews DR, Hosker JP, Rudenski AS, Naylor BA, Treacher DF. Turner RC Homeostasis model assessment: insulin resistance and beta-cell function from fasting plasma glucose and insulin concentrations in man. Diabetologia. 1985;28(7):412-9.

22. Yokono M, Takasu T, Hayashizaki Y, Mitsuoka K, Kihara R, Muramatsu Y, Miyoshi S, Tahara A, Kurosaki E, Li Q, Tomiyama H, Sasamata M, Shibasaki M, Uchiyama Y. SGLT2 selective inhibitor ipragliflozin reduces body fat mass by increasing fatty acid oxidation in high-fat diet-induced obese rats. Eur J Pharmacol. 2014;727:66-74.

23. Yamamoto C, Miyoshi H, Ono K, Sugawara H, Kameda R, Ichiyama M, Yamamoto K, Nomoto H, Nakamura A, Atsumi T. Ipragliflozin effectively reduced visceral fat in Japanese patients with type 2 diabetes under adequate diet therapy. Endocr J. 2016;63(6):589-96.

24. Chino Y, Samukawa Y, Sakai S, Nakai Y, Yamaguchi J, Nakanishi T, Tamai I. SGLT2 inhibitor lowers serum uric acid through alteration of uric acid transport activity in renal tubule by increased glycosuria. Biopharm Drug Dispos. 2014;35(7):391-404.

25. Zinman B, Wanner C, Lachin JM, Fitchett D, Bluhmki E, Hantel S, Mattheus M, Devins T, Johansen OE, Woerle HJ, Broedl UC, Inzucchi SE, EMPA-REG OUTCOME Investigators. Empagliflozin, cardiovascular outcomes, and mortality in type 2 diabetes. N Engl J Med. 2015;373(22):2117-28.

26. Giacca A, Xiao C, Oprescu AI, Carpentier AC, Lewis GF. Lipidinduced pancreatic $\beta$-cell dysfunction: focus on in vivo studies. Am J Physiol Endocrinol Metab. 2011;300(2):E255-62.

27. Unger RH. Lipotoxicity in the pathogenesis of obesity-dependent NIDDM. Genetic and clinical implications. Diabetes. 1995;44(8):863-70.

28. Lallukka S, Yki-Järvinen H. Non-alcoholic fatty liver disease and risk of type 2 diabetes. Best Pract Res Clin Endocrinol Metab. 2016;30(3):385-95. 